El Programa de Capacitación Docente Continua UNLP-ADULP como política de capacitación en servicio: análisis situacional y estrategias de mejoramiento. Mariana Lugones

Revista ES (en y sobre Educación Superior)

Vol.1, N¹-2 / Fecha de publicación: 27/12/2021

e-ISSN: 2718-6539

https://revistas.unlp.edu.ar/ES/index

IIES - Facultad de Odontología

DOI: https://doi.org/10.24215/27186539e024

\title{
EI Programa de Capacitación Docente Continua UNLP-ADULP como política de capacitación en servicio: análisis situacional y estrategias de mejoramiento.
}

The UNLP-ADULP Continuous Teacher Training Program as an in-service training policy: situational analysis and improvement strategies.

O Programa de Formação Contínua de Professores da UNLP-ADULP como política de formação em serviço: análise situacional e estratégias de melhoria.

Licenciada Mariana Lugones

Secretaria Técnico Administrativo en Especialización en Docencia Universitaria UNLP marianalugones@hotmail.com

Resumen 
En este artículo se pretende desarrollar por un lado, un análisis situacional, a partir del relevamiento de información específica sobre la implementación del Programa de Capacitación Docente Continua UNLP-ADULP, que se lleva adelante desde la Coordinación entre la Dirección de Capacitación y Docencia (dependiente de la Secretaría de Asuntos Académicos-Presidencia) y el gremio Asociación Docentes Universitarios La Plata; y por otro, proponer líneas de intervención, a partir del mencionado análisis, que posibiliten el mejoramiento del alcance y el impacto del programa.

En este sentido se propone esbozar una serie de estrategias y líneas de mejoramiento del Programa. Estas posibilitarán ampliar la información para la toma de decisiones, por un lado, y por otro, delimitar líneas de acción en el corto y mediano plazo que redundarán en una mejora del alcance e impacto del Programa.

\section{Abstract}

This article aims to develop, on the one hand, a situational analysis, based on the collection of specific information on the implementation of the UNLP-ADULP Continuous Teacher Training Program, which is carried out from the Coordination between the Training and Teaching Directorate (dependent of the Secretary of Academic Affairs-Presidency) and the La Plata University Teachers Association; and on the other, propose lines of intervention, based on the aforementioned analysis, that make it possible to improve the scope and impact of the program.

In this sense, it is proposed to outline a series of strategies and lines of improvement for the Program. These will make it possible to expand the information for decision-making, on the one hand, and on the other, to define 
lines of action in the short and medium term that will result in an improvement in the scope and impact of the Program.

\section{Resumo}

Este artigo pretende desenvolver, por um lado, uma análise situacional, a partir da recolha de informação específica sobre a implementação do Programa de Formação Contínua de Professores da UNLP-ADULP, que é efectuada a partir da Coordenação entre a Direcção de Formação e Ensino (dependente do Secretário de Assuntos Acadêmicos-Presidência) e da Associação de Professores da Universidade La Plata; e, por outro lado, propor linhas de intervenção, com base na referida análise, que permitam melhorar o âmbito e o impacto do programa.

Nesse sentido, propõe-se traçar uma série de estratégias e linhas de aprimoramento para o Programa. Isso possibilitará ampliar as informações para a tomada de decisão, por um lado, e, por outro, definir linhas de ação de curto e médio prazo que resultarão em uma melhoria no escopo e no impacto do Programa.

\section{Palabras clave}

Capacitación docente, práctica docente, posgrado, profesionalización.

\section{Keywords}

Teacher training, teaching practice, postgraduate, professionalization.

\section{Palavras chave}

Formação de professores, prática docente, pós-graduação, profissionalização. 


\section{El Programa: ámbito de gestión en que se enmarca y su caracterización.}

El Programa de Capacitación Docente Continua UNLP-ADULP se enmarca -al igual que otras estrategias de capacitación y difusión de conocimientos pedagógicos destinadas a docentes de la UNLP, tales como la carrera de posgrado Especialización en Docencia Universitaria, las Jornadas sobre las prácticas docentes en la Universidad Pública, la Revista Trayectorias Universitarias, los Cursos de Idiomas para docentes- dentro de las acciones y/o subprogramas de la Dirección de Capacitación y Docencia, dependiente de la Prosecretaría de Grado de la Secretaría de Asuntos Académicos de la UNLP. El Programa, fue asimismo presentado a fines de 2007, en el marco del denominado "Programa de Capacitación Gratuita para Docentes de Universidades Nacionales", coordinado por la Secretaría de Políticas Universitarias, y derivado de acuerdos paritarios nacionales y locales de las respectivas universidades. A través de esta política nacional, se obtiene un financiamiento sustancial para la concreción del dictado de los cursos, la compra de materiales y equipamiento tecnológico, y la coordinación administrativa de las actividades.

La coordinación general del Programa antes mencionado, y las instancias operativas que se desprenden de su implementación, son ejecutadas desde una Unidad de Coordinación bipartita constituida por integrantes de la Asociación de Docentes Universitarios La Plata y de la Dirección de Capacitación y Docencia.

Esta Dirección, tiene como funciones institucionales, promover la formación del cuerpo académico de la UNLP, a partir del desarrollo de estrategias y ámbitos formativos en los que se recuperan los lineamientos político-educativos de la 
Universidad, permitiendo el desarrollo reflexivo y crítico de la práctica docente. Asimismo, se busca la articulación con las Unidades Académicas en la producción de conocimientos sistemáticos sobre las problemáticas que atraviesan a las prácticas de enseñanza de las diferentes disciplinas, con el fin de posibilitar la configuración de líneas de innovación pedagógica y el intercambio entre los actores.

Como se refiere en el proyecto político de la UNLP, la Universidad debe ser hoy un espacio para aportar ideas y discutirlas, alejada del pensamiento único, promotora del pensamiento diverso, original y propio; caracterizada por estar llena de inquietudes, rechazos, audacias, escrúpulos y esperanzas que custodian el comportamiento ético, transparente, republicano y democrático de sus miembros. Como plantea Fernando Tauber en Pensar la Universidad “...Debe ser firmemente inclusiva y entender a la educación como una línea ininterrumpida e ininterrumpible, que no debe cortarse por un problema en el sistema que frene el proceso de toma de conocimientos de aquel que quiere seguir sumándolos, por un fracaso o deficiencia circunstancial". (Tauber, 2018) Es un compromiso de la UNLP la capacitación continua de los docentes, para el sostenimiento de la formación y promover la actualización para la mejora de la transmisión del conocimiento, así como del compromiso con la universidad pública y gratuita.

Los múltiples debates y desafíos que nos plantea este escenario encuentran al área académica de la UNLP, conformada por la Secretarías de Asuntos Académicos de la Presidencia y los equipos de gestión de las Facultades y Colegios, en un proceso de diálogo y análisis colectivo consolidado. En este Programa en particular, el diálogo es no sólo con Facultades y Colegios, sino asimismo con el gremio ADULP, y esto hace más rico el intercambio. 
Desde sus comienzos, el Programa promueve el desarrollo de actividades de postgrado y de capacitación y/o actualización no aranceladas, estructuradas en términos de Cursos y/o Seminarios de Posgrado, dirigidas a los docentes de la UNLP. A partir de una convocatoria abierta cada año, los profesores de la UNLP, expertos en diferentes temáticas, presentan proyectos de Cursos o Seminarios cuyo dictado se financia en el marco del Programa. Los cursos presentados, una vez evaluados por la Facultad afín al campo disciplinar de los mismos, son acreditados como Cursos de Posgrado o de Capacitación y se llevan adelante las acciones necesarias para su dictado. Los criterios y requisitos para realizar esta acreditación están enmarcados en la normativa de aprobación del mismo por parte del Consejo Superior de la Universidad, y expresadas en el documento Pautas Generales de Presentación y Acreditación de Proyectos en el marco del Programa, que define cuestiones relacionadas a las condiciones requeridas para presentar un proyecto.

En esta línea, el Programa de Capacitación Docente Continua UNLP-ADULP se plantea dentro de las políticas de formación e innovación de la docencia, pensadas desde la UNLP, y que resultan concordantes con las de inclusión y democratización de saberes en nuestro proyecto institucional. Las mismas se articulan en forma continua con la gremial docente a través de convenios y acuerdos paritarios con la Asociación de Docentes de la Universidad Nacional de La Plata (ADULP) y la Confederación Nacional de Docentes Universitarios (CONADU), a través del dictado en forma continua de seminarios, talleres y cursos de capacitación docente.

Resulta relevante destacar los objetivos del Programa, en tanto expresan esta intencionalidad político-institucional respecto de la formación continua en servicio de los docentes como política central del área académica de la 
universidad. Estos son:

- Garantizar el acceso de los docentes de la Universidad Nacional de La Plata a espacios de capacitación continuos que sean sostenidos como parte de un compromiso institucional mancomunado.

- Promover procesos de intercambio y reflexión conjuntos de los docentes sobre las problemáticas centrales que atraviesan hoy a las instituciones de formaciones universitarias y preuniversitarias.

- Profundizar la participación de los docentes en la vida institucional de la Universidad a partir del reconocimiento de su lugar como actores centrales de la misma.

El Programa de Capacitación Docente Continua UNLP-ADULP se estructura en dos Sub Programas básicos que contemplan las especificidades propias del espacio institucional de desarrollo, diferenciando el ámbito de los Colegios, del ámbito de pregrado y grado, teniendo en cuenta que la UNLP cuenta con docentes que se desempeñan en sus diecisiete Facultades, y en los cinco Colegios en los que se dictan los niveles inicial, primario y secundario.

A los efectos del presente escrito, se ha realizado, como punto de partida para el posterior análisis situacional, la sistematización de información referida a sus actividades, que abarca desde el año de inicio, 2007, hasta el año 2018. Del mismo surgen datos relevantes, como los expresados en la siguiente tabla en la que se puede observar la cantidad de cursos que los docentes de las distintas Unidades Académicas han dictado en estos once años de desarrollo:

\begin{tabular}{|l|l|}
\hline UNIDAD ACADÉMICA & $\begin{array}{l}\text { CANTIDAD DE CURSOS } \\
\text { DICTADOS }\end{array}$ \\
\hline Facultad de Bellas Artes (FBA) & 9 \\
\hline
\end{tabular}




\begin{tabular}{|l|r|} 
Facultad de Ciencias Agrarias y Forestales (FCAyF) & 4 \\
\hline Facultad de Ciencias Económicas (FCE) & 3 \\
\hline Facultad de Ciencias Jurídicas y Sociales (FCJyS) & 12 \\
\hline Facultad de Humanidades y Ciencias de la Educación (FAHCE) & 31 \\
\hline Facultad de Informática (FI) & 1 \\
\hline Facultad de Ingeniería (FIng) & 1 \\
\hline Facultad de Periodismo y Comunicación Social (FPyCS) & 32 \\
\hline Facultad de Psicología (FP) & 9 \\
\hline Facultad de Trabajo Social (FTS) & 1 \\
\hline Total Facultades & 103 \\
\hline
\end{tabular}

La información contenida en este cuadro da cuenta de una cuestión inicial que visibiliza la relevancia del análisis situacional, en tanto es necesario abordar la disparidad de la participación de las Facultades en el Programa, tanto para discriminar las razones de la misma como para generar líneas de acción que posibiliten un mejor aprovechamiento del mismo de parte del conjunto de la UNLP, de la misma manera, otro nudo crítico lo configura la variabilidad anual de la cantidad de cursos dictados, lo que a modo de hipótesis refiere a la distancia de los docentes respecto del interés de los temas que se abordan en la oferta anual. Es necesario intervenir en esta dimensión también, con vistas a lograr una ampliación de los docentes que se capacitan.

\section{La docencia universitaria como campo profesional. Rasgos actuales, tensiones y desafíos.}

A partir de los años 90 en la Argentina, la docencia universitaria como profesión se vio fuertemente afectada debido a las políticas de ajuste que sufrieron las universidades. En este sentido, los rasgos que se consideraban tradicionales en el imaginario colectivo acerca de esta tarea (librepensamiento, creación, 
criticidad, etc.) tuvieron que contraponerse a las nuevas lógicas: productividad, competitividad, certificación de los rendimientos. Estas nuevas características, condicionaron la identidad y el accionar docente, en un contexto dinámico de la vida universitaria. Como se ha señalado, si bien estos nuevos rasgos condicionan, no determinan la práctica docente universitaria, ni la identidad o autopercepción de los docentes respecto de su tarea; si no que se instalan tensionando posiciones ideológicas, imaginarios y prácticas en un contexto social particular donde lo hegemónico se consolida, y que además ha sido revisado en la década pasada en función de políticas públicas de corte diferente a las de tipo neoliberal de los 90'. (Morandi, 2014; Edelstein, 2014; Veiravé, 2013)

La Ley de Educación Superior (Art. 36 de los Requisitos generales), regulatoria del sistema universitario nacional, marca un aspecto fundamental en esta cuestión, al especificar que los docentes universitarios deben poseer título universitario igual o superior al nivel en el que ejercen, para poder realizar su tarea como tales. Este encuadre normativo llevó, entre otras cuestiones, a que los cursos de posgrado y las capacitaciones comenzarán a consolidarse como una demanda creciente de los docentes, especialmente en algunas instituciones y carreras.

Sumado a esta situación, aparece el denominado "credencialismo" (Collins, 1979), entendido como la compulsión y producida por la exigencia de acreditación, de certificar cursos, seminarios y capacitaciones para poder acceder a ciertos cargos, teniendo en cuenta las demandas del mercado de una acreditación especializada.

Esto tensa aún más a las instituciones educativas, cuando en un nuevo contexto, más reciente, se le suma a esta demanda de actualización, la de 
generar propuestas y proyectos democráticos de reestructuración curricular, en el marco del enfoque político de la educación superior como derecho.

\section{La capacitación en servicio como instancia de la formación docente: algunas consideraciones conceptuales.}

La capacitación docente siempre ha sido abordada como uno de los temas clave de las políticas educativas. Sea frente a la tan mentada "crisis" de la educación, o al avance de las nuevas tecnologías y al estallido de la información, los docentes estuvieron y están siendo considerados como una población que se "debe poner al día".

En cuanto al término "Capacitación", según la RAE capacitar es "hacer a alguien apto, habilitarlo para algo". En el Diccionario de Ciencias de la Educación, se lo define como un dispositivo que permite la adquisición de nuevas tácticas y estrategias, y se lo vincula con la actualización ante el carácter vertiginoso de nuevos conocimientos. El perfeccionamiento o la capacitación debe ligarse a “(...) lo que está bien, lo que de alguna manera goza de amplia y sostenida aprobación), esto puede ser una práctica o un programa, etc.” (Squella 1998 en Villarroel, 1998: 25). Según Pérez Gómez (1992) la formación continua puede considerarse como un proceso que posibilitaría la modificación permanente de las ideas, las actitudes y las actuaciones, incitando a la discrepancia, al cambio y a la generación de nuevos aprendizajes, gracias al aporte de los diferentes campos de las disciplinas.

En palabras de Vezub "Esta manera de pensar la formación permanente de los docentes (...) generó una concepción instrumental de la formación continua donde subyace la idea de un maestro que carece, un sujeto deficitario, ineficaz que debe ser reconvertido. La capacitación se dirigió a suplir las lagunas de la 
formación y compensar ineficiencias..." (2009:10)

La práctica docente, como práctica social, es inevitablemente compleja. Complejidad que implica múltiples determinantes y contradicciones. La práctica docente tenida en cuenta como parte de un proyecto pedagógico, como apuesta ética, que no se puede desconocer. "Lo que falta no son saberes procedimentales complementarios, sino una práctica sistemática de reflexión sobre lo que va pasando con una alternancia -de fuerte articulación y no yuxtaposición- de tiempos de clase y tiempos de trabajo en grupos de formación, lo que plantea la necesidad de incorporar nuevos sentidos" (Edelstein, 2011:111) Así, la capacitación docente puede y debe ser pensada desde un lugar de docente muy distinto al modelo hegemónico, desde un espacio de reflexión intencional que produzca nuevas alternativas, diferentes y críticas.

La capacitación de los profesores -como parte de su formación permanente, sea ésta pedagógica o no- se convierte en una condición necesaria para poner en marcha una renovación en sus tareas. En este sentido, los programas de capacitación son estrategias que tienen como finalidad mejorar las prácticas de la enseñanza

La formación docente, al decir de Azucena Rodríguez Ousset (1994) implica una capacitación en el campo del saber y una capacitación pedagógica; tiene por tanto un doble carácter. Toda formación implica una revisión y una crisis, abrirse a nuevas formas de pensar, "escuchar otras voces", incorporar el intercambio interdisciplinar. El problema de los cursos de formación y de capacitación puede ser éste, el de no generar estos procesos de revisión crítica, si no estancar el saber y no conmover las prácticas docentes. 


\section{Las valoraciones de los docentes sobre sus prácticas y su identidad profesional.}

Los cursos de este Programa se han pensado siempre desde un espacio interdisciplinario en el que se reflexione colectivamente sobre estos contextos y realidades que interpelan a los docentes desde las prácticas. Con la intención de que ese análisis nos permita comprender los condicionantes de la acción docente, al mismo tiempo, que posicionarnos en la construcción colectiva de su transformación.

Las prácticas docentes se comprenden con una perspectiva socio-histórica. Historicidad que no deja huella solo en los condicionantes materiales e institucionales que las configuran y en los modos de regulación y producción del trabajo docente en toda su diversidad y complejidad, sino también en las construcciones subjetivas que conforman miradas compartidas que se han ido construyendo en la trayectoria universitaria como docentes y estudiantes (Edelstein, 2014)

\section{Hacia la construcción de un análisis situacional del Programa}

De los cursos de posgrado dictados en los once años que lleva desarrollándose el Programa (2007-2018) se puede observar que la Facultad de Humanidades y Ciencias de la Educación, así como la de Facultad de Periodismo y Comunicación Social, se presentan como las Unidades Académicas (en adelante UA) cuyos campos temáticos, cuentan con mayor incidencia en la presentación y puesta en práctica, por parte de los formadores, de cursos destinados a docentes de grado (31 y 32 respectivamente), mientras que le siguen propuestas aprobadas por la Facultad de Ciencias Jurídicas, con 12 cursos dictados, luego las de Psicología y Artes, con 9 cursos cada una. En 
relación con lo antes mencionado podría pensarse a modo de hipótesis que, a excepción de la Facultad de Ciencias Jurídicas y Sociales, las restantes que han aprobado la mayor cantidad de cursos son Facultades que poseen carreras de formación docente (Profesorados), y que por ende, producen saberes sobre la enseñanza, desarrollan un campo académico ligado a la formación de docentes, y cuentan asimismo, con recursos formados que se constituyen como formadores en el marco del programa, en proyectos para la capacitación continua de otros docentes.

Otras docentes que han presentado proyectos dentro del Programa pertenecen a las Facultades de Ciencias Agrarias (6), Ciencias Económicas, (3), mientras que Trabajo Social, Ingeniería e Informática han dictado (1) curso en el periodo analizado. Esto genera que los enseñantes opten por seguir capacitándose en su ámbito laboral, de modo que esta dinámica que bien se encarga de fomentar la capacitación en su equipo docente no favorece la puesta en diálogo de las distintas disciplinas que conforman el ámbito universitario, como se propone desde el Programa. Respecto de estos datos, cabe señalar, por un lado, la existencia de acciones de formación docente desarrolladas por la propia Unidad Académica y, en algunos casos por sus Unidades Pedagógicas, entre las que se encuentran las facultades de Arquitectura, Ciencias Agrarias y Forestales, Ciencias Económicas, Ciencias Exactas, Odontología, y Ciencias Jurídicas y Sociales. En menor medida esto ocurre con las Facultades de Astronómicas y Geofísica, Ciencias Veterinarias y Ciencias Médicas, por lo que, como línea de acción de este análisis se evidencia la necesidad de indagar o identificar desde el Programa de capacitación docente continua UNLP-ADULP, la necesidad de capacitación de los docentes de las Facultades mencionadas. 
En cuanto a las áreas, hay una predominancia en el interés por el cursado de propuestas que abordan problemáticas pedagógico-didácticas de la docencia universitaria (49 de los 91 cursos dictados). Si tomamos como parte de esta área los 13 cursos que abordan las nuevas tecnologías y educación a distancia en la enseñanza universitaria, y a los 6 cursos que se enmarcan en las prácticas docentes desde las perspectivas de las didácticas especiales 0 específicas, el énfasis en estos temas se evidencia claramente. Del resto de las áreas, 19 de los cursos dictados refieren a epistemología de las ciencias y metodología de la investigación en campos disciplinares específicos, 12 abordan el área referida a universidad, sociedad y políticas públicas y proyectos de extensión, y el resto se refieren a problemáticas específicas inherentes a algún campo disciplinar. Podemos deducir que a la hora de elegir presentar un proyecto para dictar y/o realizar un curso de capacitación, los docentes optan por problematizar la docencia universitaria desde una dimensión pedagógico-didáctica. Sin embargo, no se puede pasar por alto el gran número de cursos que proponen comprender la especificidad en la que se encuentran trabajando, ya sea desde la práctica en el aula o la investigación, generando un marco de intercambio amplio y complejo.

También es importante volver a resaltar que, en años recientes de desarrollo del Programa, se han presentado una cantidad importante de cursos referidos a las nuevas tecnologías, una temática transversal a todas las áreas de conocimiento que genera interés tanto desde los docentes de grado como de sus alumnos. Uno de los desafíos de la educación superior hoy es, justamente, analizar cómo las innovaciones tecnológicas modifican o transforman la relación pedagógica entre educadores y educandos, y posibilitan otras formas de acceso a los saberes. Resulta también interesante remarcar el interés de los 
docentes por dictar cursos relacionados con la temática vinculada a las políticas públicas y la Universidad, en tanto, desde 2012 a 2018 se ha incrementado este número representando la importancia y la necesidad de trabajar interdisciplinariamente sobre esta temática, se duplicó prácticamente. Si bien podemos identificar el 2008 como el año de mayor cantidad de cursos dictados (15), y mayor cantidad de docentes capacitados (166 docentes), debemos señalar que, a excepción de los años 2015, en que se dictaron 13 cursos y 2017, con 11 cursos; en los años restantes, se ha sostenido un número similar, tanto en cantidad de cursos dictados, como docentes capacitados; estando esta cantidad en un número de entre 6 y 8 el promedio de cursos ofrecidos por el Programa. Cada año el número de docentes que se inscribieron en los cursos se mantiene en alrededor de 60. Este dato, si bien arroja una cierta estabilidad, en el promedio de cursos marca una importante línea a indagar, pensando en la diferencia que existe cada año, entre la cantidad de cursos efectivamente dictados con la de proyectos presentados, en donde la primera es menor que la segunda. Se trata de cursos que cumplen con los requisitos del programa, que han sido habilitados por las Facultades, pero que no logran reunir un número mínimo de inscriptos para ponerse en funcionamiento. Esta cuestión evidencia un aspecto a mejorar en el Programa, lo que puede lograrse eventualmente a partir del rastreo que aquí se propone de las demandas de capacitación de los docentes de la UNLP.

Construcción de líneas de intervención para el mejoramiento del Programa a partir del análisis situacional

A partir del relevamiento y análisis inicial efectuado del Programa de 
Capacitación Docente Continua UNLP-ADULP que comprende, como ya se ha señalado, desde el año 2007, de inicio del mismo, al año 2018, se propone esbozar una serie de estrategias y líneas de mejoramiento del mismo. Estas posibilitarán ampliar la información para la toma de decisiones, por un lado, y por otro, delimitar líneas de acción en el corto y mediano plazo que redundarán en una mejora del alcance e impacto del Programa.

En este sentido se proponen las siguientes líneas de acción tendientes a lograr estos objetivos.

\section{- Relevamiento de demandas de capacitación continua}

En una primera etapa, y con el propósito de alcanzar uno de los objetivos específicos de este Trabajo centrado en ampliar las líneas temáticas del Programa, se realizará un relevamiento de los intereses de los docentes, a partir de la base de datos acumulada de los docentes cursantes tomando una muestra de cada año de su desarrollo.

Se buscará relevar temáticas de interés de los docentes, así como preferencia de modalidad de cursada.

\section{- Instrumentos de evaluación continua}

Se instrumentará una encuesta de valoración de docentes cursantes, de las actividades al finalizar el desarrollo de cada curso, haciéndolo de manera sistemática mediante una encuesta en línea, que permita poner en evidencia los aportes, así como las dificultades que puedan aparecer en el transcurso del cuatrimestre.

También se tomarán encuestas a los docentes dictantes, a modo de relevamiento de las facilidades o dificultades que pudiesen haber aparecido en todo el proceso desde la presentación del proyecto hasta el dictado del curso.

En esta etapa también se realizarán entrevistas semiestructuradas a una 
muestra de docentes, tanto cursantes como dictantes, en las que se explorará sobre cuestiones relacionadas tanto con la organización como con el desarrollo de los cursos. Estas entrevistas permitirán ahondar más con cada docente sobre las particularidades del curso que dictó o cursó, teniendo en cuenta la Facultad a la que pertenece y el área temática desarrollada. Para esto, la muestra será tomada con un representante de cada Unidad Académica por curso. Esto permitirá que los datos recabados sean más heterogéneos y significativos.

Con estos datos cuali y cuantitativos sistematizados, junto con el análisis de los datos recabados en las encuestas y entrevistas, se organizará un Informe Anual del Programa de Capacitación docente continua UNLP-ADULP, que se publicará. El objetivo de este Informe será mejorar el funcionamiento del Programa, incentivar la presentación de nuevas propuestas, motivar a los docentes de las distintas Unidades Académicas a participar de los cursos, comunicarse de manera más efectiva entre los docentes y la Coordinación del Programa.

\section{- Mejoramiento de estrategias de las acciones de coordinación y comunicación con Facultades y Colegios}

En una segunda etapa se propondrán estrategias que incentiven la presentación de proyectos para el pregrado, en ese sentido se organizarán reuniones con las autoridades de cada Colegio de la UNLP, con el objetivo de recabar temáticas de interés para sus docentes. Estas reuniones se podrán ampliar también a los Secretarios Académicos de las distintas Facultades, con la misma intención. Poder tener un relevamiento de información sobre las áreas temáticas de interés, así como la fluidez de la comunicación con el Programa. También esto buscará incentivar la presentación de Proyectos en las 
convocatorias anuales de todas las Facultades.

- Desarrollo de propuestas de formación no presencial

Otra línea interesante refiere a la posibilidad de incluir mayor cantidad de cursos totalmente virtuales, que no requieran la presencialidad. En este sentido se podría utilizar la plataforma Aulasweb de la UNLP, como soporte tecnológico. Así como las tutorías de la Dirección general de educación a distancia, en caso de ser necesaria una capacitación específica para los docentes dictantes.

\section{- Ampliación de los destinatarios del Programa}

Como otra etapa dentro de este Proyecto, se podrá pensar en la posibilidad de incluir dentro de los cursantes, a los becarios y adscriptos, ya que estos cumplen una función docente aún en su etapa formativa. Y este espacio podría ser interesante con su inclusión y sus aportes. Esta necesidad surge de varias consultas que han sido realizadas durante los años de ejecución de este Programa.

\section{- Revisión de procedimientos administrativos}

Reformular algunos procesos administrativos que faciliten y efectivicen la presentación de proyectos en las convocatorias anuales, tales como la modificación de las Pautas de presentación, de modo que no sea necesaria la copia en papel, sólo alcance con la presentación en Mesa de entradas y enviar por mail la copia digital.

\section{Reflexiones Finales}

Luego de este recorrido en torno de los procesos involucrados en la gestión del Programa de Capacitación Docente Continua UNLP-ADULP, desde el año 2007 hasta el 2018, y teniendo en cuenta que los Programas de capacitación y 
actualización son vehículos indispensables para renovar y perfeccionar el ejercicio docente, ha sido de gran importancia esta sistematización, ya que proveyó de herramientas relevantes para continuar con la tarea de coordinación del Programa, así como pensar en estrategias de mejoramiento del mismo.

El análisis de tales programas es aconsejable para corroborar su ejecución, utilidad y trascendencia, así como para compartir dentro de la institución (en este caso la Universidad Nacional de La Plata) los procedimientos y resultados de estos Programas en la planta docente, así como su impacto en cada una de las Unidades Académicas.

En este sentido, pensar a la reflexión como proceso que interpela a la propia práctica docente, teniendo en cuenta las respuestas de los docentes, tanto cursantes como dictantes, de las encuestas y de las entrevistas, permitiría organizar líneas de acción que tiendan a ampliar la propuesta de cursos y seminarios. Pensar en la reflexión para una mejor propuesta dentro del Programa de capacitación docente continua UNLP-ADULP.

Asimismo, poder delinear mejoras de las prácticas docentes en pos de un trabajo político-pedagógico, comprometido con un proyecto educativo que sea inclusivo y contenedor de las necesidades de los docentes. Teniendo como base una continuidad de las políticas públicas para la capacitación docente.

Otro discurrir después realizado este análisis, aparece como la importancia de que los docentes que participan de este Programa de capacitación puedan transmitir en sus cátedras lo aprendido, lo interiorizado, así como la importancia del intercambio con otros, de otras disciplinas y de otras carreras.

Tomando las palabras de Matus, quien plantea que planificar es como pensar antes de actuar, pensar con método, de manera sistemática, pensar ventajas y 
desventajas de tales o cuales objetivos. Como punto de partida, para pensar, para preguntar y repreguntar-se, para "acomodarse" al contexto, abrir-me a preguntas, repensar lo planificado. Re-planificar. Escuchar a los docentes, sus demandas, necesidades, intereses, devoluciones. Tener en cuenta la incertidumbre de situaciones cotidianas, nuevos espacios, nuevas formas de enseñar, nuevos posicionamientos docentes. Acompañar como Universidad en todo ese proceso que implica a la práctica docente.

\section{Bibliografía}

- A.A.V.V. (2000) Diccionario de las ciencias de la educación, Madrid, Santillana.

- Collins, R. (1989) La sociedad credencialista. Sociología histórica de la educación y la estratificación, Madrid, España. Ediciones Akal.

- Diccionario de la Real Academia Española. https://www.rae.es/

- Duschatzky L. (2014) De la capacitación al acompañamiento. I Encuentro Internacional de Educación Espacios de investigación y divulgación. NEES - Facultad de Ciencias Humanas - UNCPBA. Tandil, Argentina.

- Edelstein, G. (2011). Formar y formarse en la enseñanza. Cap. 4 Buenos Aires, Paidós. Cuestiones de la Educación.

- Edelstein, G (2014) Formar-se para la enseñanza en las universidades. Aportes desde experiencias de posgrado. En: Morandi, G. y Ungaro, A. La experiencia interpelada. Prácticas y perspectivas en la formación docente universitaria, La Plata, Argentina, Edulp.

- Giordano, C. (2009) 23 Tesis sobre la Tesis. La comunicación para la transformación de la gestión educativa. Tesis de Doctorado. Cap. 8. 
- Matus, C. (1987) Política, planificación y gobierno. Fundación Altadir. Caracas.

- Morandi, G. (2014) El campo de la formación docente de profesores universitarios: configuración histórica y perspectivas políticoacadémicas. En: Morandi, G. y Ungaro, A. La experiencia interpelada. Prácticas y perspectivas en la formación docente universitaria, La Plata, Argentina, Edulp.

- Plan Estratégico Gestión 2014-2018 de la Universidad Nacional de La Plata. Publicación institucional de la Universidad Nacional de La Plata.

- Pérez Gómez, A. (1992) “Enseñanza para la comprensión”, en: Gimeno Sacristán, J y A. Pérez Gómez Comprender y transformar la enseñanza, Madrid, Morata.

- Rodríguez Ousset, A. (1994) Problemas, desafíos y mitos en la formación docente. En Perfiles educativos Número 63, México.

Disponible en https://www.iisue.unam.mx/perfiles/index.php?numero=63\&anio=1994

- Tauber, F. (2008) Tesis de Doctorado "La comunicación en la planificación y gestión para el desarrollo de las instituciones universitarias públicas argentinas: el caso de la Universidad Nacional de La Plata en el trienio junio 2004-mayo 2007".

- Tauber, F. (2018). Pensar la Universidad. Proyecto institucional de la Universidad Nacional de La Plata-2018-2022. Publicación institucional de la Universidad Nacional de La Plata.

- Veiravé, M. (2013). La formación docente universitaria. Los posgrados en educación superior, una lectura desde los proyectos de Ley de Educación Superior en Argentina. Revista RAES, (6), 16 - 
26.http://www.revistaraes.net/revistas/raes6 conf2.pdf

- Vezub, L. (2009) Notas para pensar una genealogía de la formación permanente del profesorado en la Argentina. En Revista mexicana de investigación educativa.Vol.14 no.42. México. Disponible en línea: http://www.scielo.org.mx/scielo.php?script=sci arttext\&pid=S1405$\underline{66662009000300014}$ 